[30] Butterman, M., Tietz, D., Orban, L. and Chrambach, A., Electrophoresis 1988, 9, 293-298.

[31] Tietz, D., Gottlieb, M. H., Fawcett, J. S. and Chrambach, A., Electrophoresis 1986, 7, 217-220.
1321 Orban, L., Tietz, D. and Chrambach, A., Electrophoresis 1987, 8 , 471-476.

133] Stellwagen, N. C., Biochemistry 1983, 22, 6186-6190.

134] Peacock, A.C. and Dingman, C. W., Biochemistry 1968,7,668-673.
Stephan Diekmann

Max-Planck-Institut fuir

Biophysikalische Chemie, Göttingen

\section{The migration anomaly of DNA fragments in polyacrylamide gels allows the detection of small sequence-specific DNA structure variations}

\begin{abstract}
Curved DNA fragments have a reduced electrophoretic mobility in polyacrylamide gels. The retardation in gels is extremely sensitive to small structural variations which influence the DNA helix axis. This gel assay can also be used to detect very small structural variations in DNA sequences which are not curved: The noncurved seguences of interest can be combined with curved stretches in phase with the helix turn. Using such sequence constructions, even subtle influences on the DNA helix axis can be detected. Experiments of this kind allow the determination of a relative order of sequence-specific DNA twist and wedge angles.
\end{abstract}

\section{Introduction}

The local structure of the DNA double helix is modulated by the sequence of the molecule [1]. These sequence-specific structure modulations can also influence the path of the helix axis in space. If $\mathrm{dA}_{n}$ tracts are present in the molecule with $n \geq$ 2 , the helix axis is no longer straight. This effect becomes large when the $\mathrm{dA}_{\mathrm{n}}$ tracts are phased with the turn of the helix (for reviews see $[2,3]$ ). The nonlinearity of the DNA helix axis (i.e. the curvature of the molecule) can be detected using several methods: circularization of DNA molecules $[4,5]$, electron microscopy $[6,7]$, and gel migration anomaly [8-15]. Results obtained from these measurements can be compared with DNA structures obtained from NMR [16] and crystallographic $[17,18]$ analysis. DNA molecules having a curved helix axis migrate slower through the acrylamide gel pores than straight molecules, resulting in a migration anomaly. This effect has been used to determine properties of DNA molecules containing $\mathrm{dA}_{\mathrm{n}}$ sequences. Since a quantitative theory for the migration of DNA molecules through gel pores explaining the migration anomaly of curved fragments does not exist, the migration anomaly is calibrated relative to the mobility of normal marker fragments [1.9]. The extent of the gel migration anomaly of curved DNA fragments defined in this way in polyacrylamide gels shows roughly a square dependence on the degree of curvature of the molecules [20, 21]. Recently, models have been presented which relate the gel migration anomaly to sequence-specific DNA parameters $[22,23]$.

Correspondence: Dr. Stephan Diekmann, Max-Planck-Institut für Bio physikalische Chemie, Abteilung Molekulare Biologie, Am Faßberg, D-3400 Göttingen, Federal Republic of Germany

Abbreviations: bp, base pairs; TBE, Tris-boric acid-EDTA buffer
Absolute values for structural DNA parameters are not obtained, which is a principal drawback of the gel assay. Therefore, conclusions obtained from this technique should be checked by other methods based upon better understood physical techniques. However, rigorous techniques are often elaborate and unable to detect the small structural variations which can be resolved in the gel (see for example [24]). Experimentally, the gel assay is convenient. Small amounts (a few nanograms) of DNA can be analyzed. Furthermore, the mobility of DNA molecules in gels is not affected by the presence of other DNA fragments or a variety of chemical compounds when present in small quantities. The gel migration anomaly of considerably curved DNA fragments is a rather large effect since the anomaly increases with the square of the degree of the curvature $[20,21]$ so that the experimental results are easy to analyze.

The migration anomaly has been measured to determine structural properties of $\mathrm{dA}_{\mathrm{n}}$ tracts. However, this technique can also be used to measure sequence-specific structural influences of normally straight DNA sequences. In particular, the method can be applied to determine relative twist or wedge angles of DNA sequences. The uncurved stretches do not result in a migration anomaly and the gel matrix is insensitive to small variations of the DNA structure in linear molecules. Therefore, to allow the gel assay to be applied, these sequences of interest should be combined with curved sequences in such a way that a considerable migration anomaly is obtained. Then the anomaly of the curved sequences is modulated by the structural variations of the linear stretches of interest. This approach uses the extreme sensitivity of the gel migration anomaly to detect structural influences on the helix axis and sequence-specific variations thereof. Temperature and salt influences on the sequence-specific structural modulations can also be measured. 


\section{Materials and methods}

\subsection{Oligonucleotide synthesis}

DNA oligonucleotides were synthesized on an Applied Biosystems (380 B DNA) synthesizer. The oligonucloetides were purified twice by reverse phase high performance liquid chromatography (HPLC) according to Ott and Eckstein [25]. First, the oligonucloetides were purified while they still had their chemical protection group; then the protection group was removed and the oligonucleotides were purified again. Oligonucleotides prepared in this way could be ligated to high multimer numbers and gave sharp bands in polyacrylamide gels. If this purification routine is simplified and only the second or no HPLC purification is carried out, additional bands might be observed in the ligation multimer distribution. Furthermore, the distribution might lack high multimer numbers and the bands in the polyacrylamide gels may sometimes become fuzzy. The oligonucleotide sequence can be easily verified. After phosphorylation by polynucleotide kinase, annealing and ligation, the multimer distribution can be radioactively labelled and chemically sequenced according to the protocol of Maxam and Gilbert [26].

\subsection{Polyacrylamide gels}

Fresh acrylamide solution (29:1 acrylamide to $N, N^{\prime}$-methyl enebisacrylamide) in $0.5 \times \mathrm{TBE}$ buffer, containing $45 \mathrm{~mm}$ Tris (trishydroxymethylaminomethane), $45 \mathrm{~mm}$ boric acid, 1.25 mM EDTA (ethylenedinitrilotetraacetic acid disodium salt dihydrate) and ammonium peroxodisulfate is filtered, mixed and degassed carefully. After addition of $N, N, N^{\prime}, N^{\prime}$-tetramethylethylenediamine (TEMED), the solution is poured between glass plates. The gel glass plates used here are $16 \mathrm{~cm} \times$ $22 \mathrm{~cm}$ in size, with $1.5 \mathrm{~mm}$ spacings. The comb and a platinum $(\mathrm{Pt})$ temperature detector are placed in the acrylamide solution between the glass plates. The polymerization is allowed to proceed for several hours. Finally, the comb is removed and the wells are carefully washed with buffer. The glass plates including the gel and the temperature monitor (see below) are placed into a gel tank, the upper electrolyte compartment of which is of normal size. The lower compartment is large $(6 \mathrm{~L})$ and embeds the whole gel and the upper electrode compartment. Thermostated water runs in glass helices through both compartments, thermostating the entire isolated gel tank. The gel is prerun for several hours in $0.5 \times \mathrm{TBE}$ running buffer at $4.5 \mathrm{~V} / \mathrm{cm}$ until the temperature in the gel and the electrical current are constant. The DNA solutions are mixed with Ficoll and tracking dyes to a total volume of about $5 \mu \mathrm{L}$ and loaded on the gel at the bottom of the wells. After the run (of several hours) the gel is stained in an ethidium bromide solution ( $\mathrm{ca} .2$ $\mu \mathrm{g} / \mathrm{mL}$ ) and washed in water for $1 \mathrm{~h}$ each. The gel is then placed on a $254 \mathrm{~nm}$ UV transilluminator and photographed through a set of 3 optical filters (UV, yellow, orange) using a high resolution black and white film (Kodak AHU; this film should be processed with "Neofin blue" developer at $30^{\circ} \mathrm{C}$ for $4 \mathrm{~min}$ ). The film negative is enlarged to at least the original gel size $(16 \mathrm{~cm} \times 22 \mathrm{~cm})$ and analyzed. A typical gel is shown in Fig. 1.

The migration anomaly is temperature- and salt-dependent. For example, a change in migration anomaly was already detected upon removal of EDTA from the running buffer without addition of salt [27]. Therefore, these external condi-

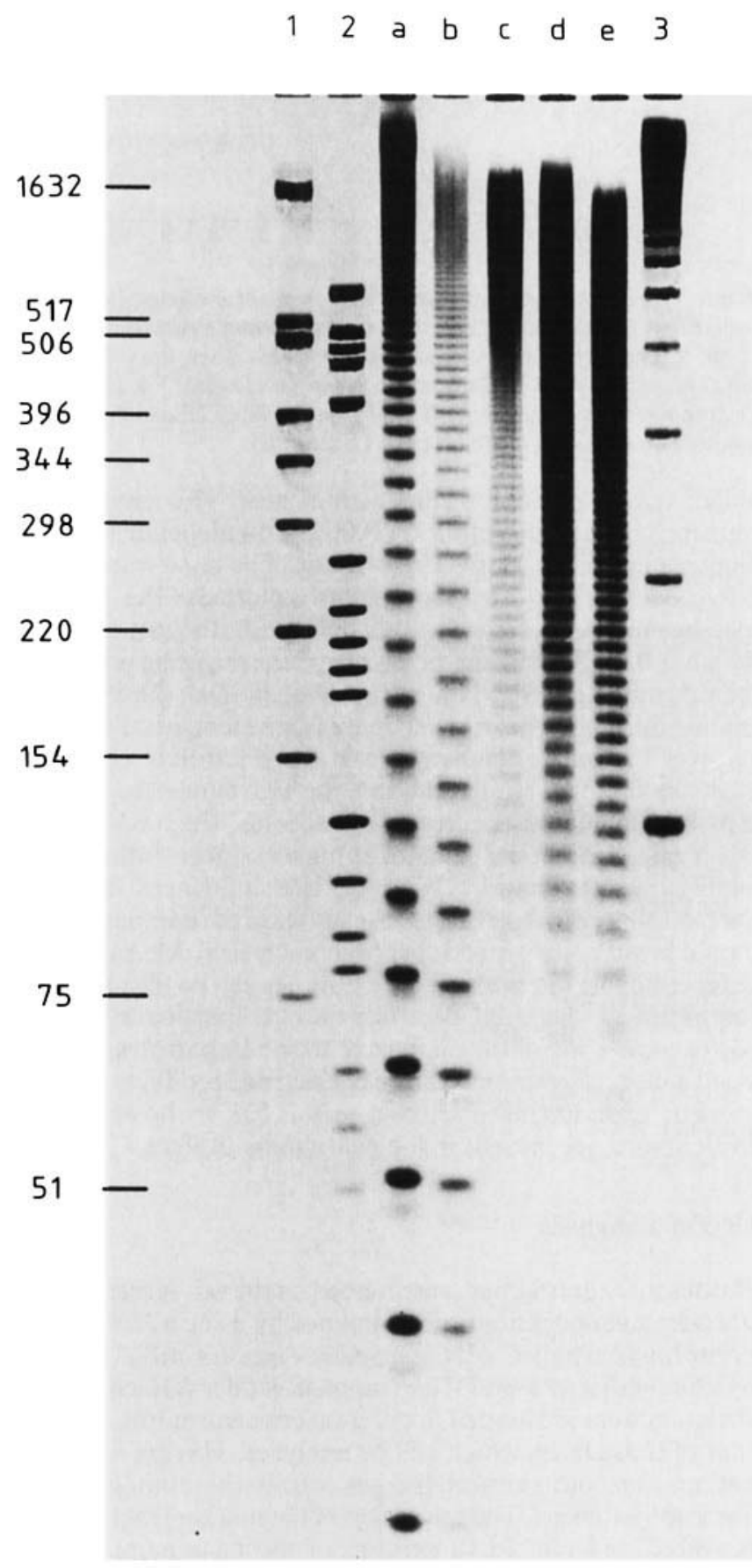

Figure 1 . A $10 \%$ polyacrylamide gel run in $0.5 \times \mathrm{TBE}$ buffer at room temperature and $4.5 \mathrm{~V} / \mathrm{cm}$. DNA marker fragments of restriction digests of pBR322 [28] are run in lanes (1) digest with HinfI and (2) digest with HaeIII. In lane (3) a 123 bpladder (BRL) is electrophoresed. Lanes (a) to (e) contain ligation ladders of the sequences $5^{\prime}-\mathrm{d}(\mathrm{GGGCAAXAAC}) * 5^{\prime}-\mathrm{d}(\mathrm{C}-$ CCGTTYTTG) with the base pair $d X * d Y$ being (a) $d A * d T$, (b) $d I * d C$, (c) dI*dT, (d) desoxy-2-aminopurine*dT, and (e) desoxy-2,6-diaminopurine*dT (see $[36]$ ).

tions are monitored. When the salt dependence of the migration anomaly was measured, additional salt was added to the running buffer as well as to the acrylamide solution before polymerization. The conductivity of the buffer is measured by a Wayne-Kerr bridge before and after the gel run. The temperature in the gel is measured during the run by a Pt element polymerized in the gel. The resistance read-out is temperature-calibrated and compared to a preset electrical 


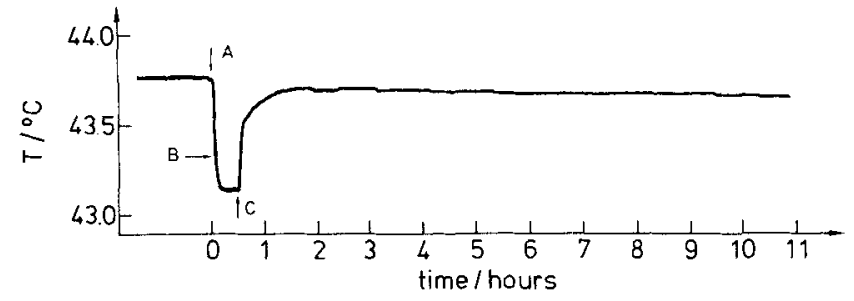

Figure 2. Temperature measured during the course of an electrophoresis experiment. A $6 \%$ poly acrylamide gel was preelectrophoresed several hours at $150 \mathrm{~V}$ until temperature and current were stable. Then, the voltage was disconnected (at point $A$ ) and the DNA samples loaded. The DNA was electrophoresed into the gel at $50 \mathrm{~V}$ (at point B). After $25 \mathrm{~min}$ the voltage was increased back to $150 \mathrm{~V}$ (at point $\mathrm{C}$; see [37]).

value; only a difference value is indicated. This enables extremely sensitive detection $\left( \pm 0.002{ }^{\circ} \mathrm{C}\right)$ independent of the temperature band width of the device. The temperature in a $6 \%$ gel during an electrophoretic run is plotted in Fig. 2. After loading, the temperature in this gel during the run is stable within $\pm 0.03{ }^{\circ} \mathrm{C}$. In general, divalent ions might be present in the gel running buffer. Due to the divalent ions, the electrical heating during the gel run can increase the temperature in the gel over the buffer temperature in the electrolyte compartments by up to $10^{\circ} \mathrm{C}$. In addition, the high current (up to 100 $\mathrm{mA}$ ) is combined with electrode processes which reduce the DNA migration in the gel and, at higher concentrations, will modify the gel matrix. To avoid the influences of these electrode processes on the gel, the buffer in the compartments should be pumped with $5 \mathrm{~L}$ per hour each way. Alternatively, semipermeable ion exchange membranes can be placed in the compartments between electrode and gel (particularly a cation exchange membrane in the cathode compartment). Such membranes stop the penetration of electrode products into the gel. For experiments discussed in this paper, however, no divalent ions are present in the gel running buffers.

\subsection{Data analysis}

Various marker fragments are loaded on the gel. A reasonable DNA fragment distribution is obtained by using a HaeIII and a HinfI + (partial) EcoRI restriction digest of pBR322 [28] together with a 123 bp DNA fragment ladder (Gibco-BRL). These markers are loaded in different concentrations on both sides of those lanes which will be analyzed. Having identical markers on both sides of the gel checks the homogeneous migration in the gel. The migration of the marker fragments is measured and plotted (logarithm of the base pairs vs. $\mathrm{cm}$ migrated; Fig. 3). This graph is the calibration for the length determination of the multimer distributions. The graph is only roughly linear and not the linear approximation, the real curve is taken for the length determination. Since short oligonucleotides with less than 50 base pairs (bp) migrate according to their size independent of any curvature, the short oligonucleotides in the multimer distribution are used to identify the sequence length of the molecules. The apparent length of the DNA fragment divided by the actual sequence length defines the $k$-factor which is plotted versus the actual fragment sequence length given in base pairs or number of oligonucleotide repeats. Since a quantitative physical theory does not yet exist, explaining the migration of curved DNA molecules through gels, the migration anomaly of curved DNA fragments must be measured relative to the "normal" marker fragments. The only gel mobilities which are interpreted and discussed are obtained from neighboring lanes in the same gel.

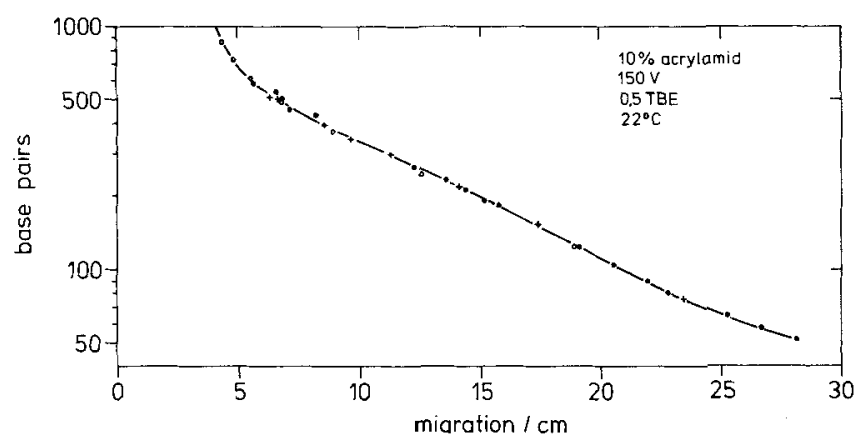

Figure 3. Reference curve of the marker fragments in a $10 \%$ polyacrylamide gel at $22^{\circ} \mathrm{C}$ in $0.5 \times \mathrm{TBE}$ buffer and $4.5 \mathrm{~V} / \mathrm{cm}$. The graph shows a pBR322 [28] restriction digest with Hinf $(+)$, HaeIII (O), and a 123 bp ladder $(\mathrm{O})$

\section{Results and discussion}

\subsection{DNA twist variations}

If ligated multimer distributions of curved oligonucleotides with 10 or $11 \mathrm{bp}$ length are analyzed, the relative migration anomaly ( $k$-factor) levels off at about $200 \mathrm{bp}$. Two different hypotheses might explain this plateau: (i) either the gel matrix does not detect DNA curvature beyond the persistence length of the molecule (under the gel buffer salt conditions about 150 $b p$ ), or (ii) the phasing between helix and sequence repeat is slightly violated, leading to a not perfectly planar curvature of the molecule [22]. If $21 \mathrm{bp}$ long molecules of similar sequence as the 10 and 11 mers are constructed (thus having a $10.5 \mathrm{bp}$ sequence repeat), the migration anomaly of the multimer distribution does not level off at $200 \mathrm{bp}$; instead the anomaly continues to increase well beyond 300 bp length [29] (Fig. 4). This finding directly contradicts the flexibility argument (i): If the leveling-off were due to the global sequence-unspecific and isotropic DNA flexibility, the effect would be length-dependent but independent of the small changes in the repeat length. Thus, the migration anomaly of the $21 \mathrm{bp}$ long multimers should also level off at $200 \mathrm{bp}$ in the same way as the 10 and 11 bp multimers, in clear contrast to the experimental finding. On the other hand, if the leveling-off is due to a phasing violation, the effect should indeed be sensitive to small changes in the sequence repeat (while the helical repeat is kept nearly constant) as found experimentally.

Obviously, the migration anomaly of the 10 and $11 \mathrm{bp}$ multimers levels off at about $200 \mathrm{bp}$ because the sequence repeat is not in perfect phase with the helical repeat. The helical repeat is determined by the sequential dinucleotide twist angles of the sequence. Above, very similar DNA sequences with different sequence repeat lengths were compared. Now, we instead keep the sequence repeat length constant and slightly modify the DNA sequence. This will result in a modified helical repeat. The different helical repeat (due to the different sum of the dinucleotide twist angles) will have an influence on the leveling-off of the migration anomaly of these sequences. Thus, by analyzing the leveling-off behavior of sequence-related multimer distributions we can observe the effects of different sequence-specific DNA twist angles. For such experiments, double-stranded oligonucleotides are constructed, containing the same curvature inducing sequence (for example a $\mathrm{dA}_{5}$ tract) and each being 10 or $11 \mathrm{bplong}$, thus having a small but not negligible difference between helical and sequence repeat. 


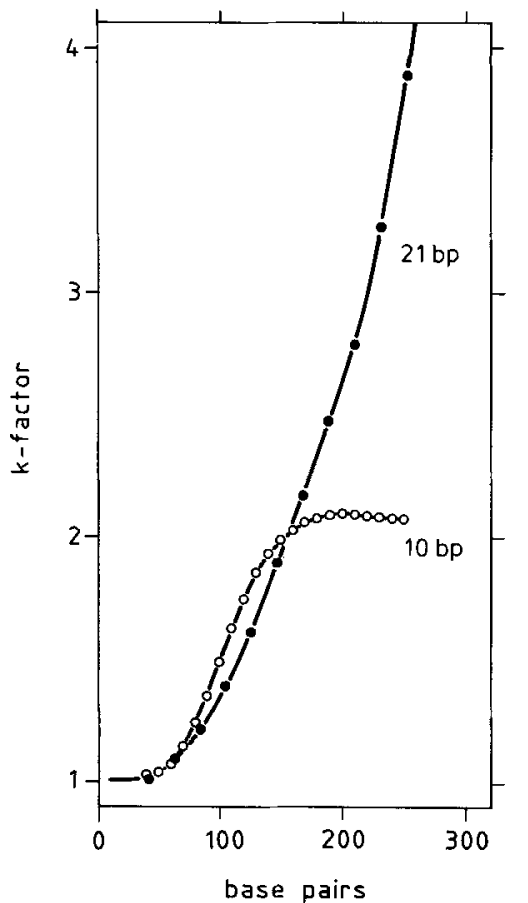

Figure 4. Apparent length in the gel divided by the sequence length ( $k$-factor) versus the bp number in the multimer distribution of the sequences $5^{\prime}$ dCAAAAACCGTGCAAAACTCGA-3' (21 bp repeat length, $\bullet$ ) and $5^{\prime}$-dGGGCAAAAAC $-3^{\prime}(10 \mathrm{bp}$ repeat length, $O)$. The $k$-factor is determined in $10 \%$ polyacrylamide gels with HaeIII and Hinf $\mathrm{L}$ digests of the plasmid pBR 322 [28] as length markers. The experimental error of the $k$-factor is \pm 0.02 (see [29]).

Only in the sequence outside the $\mathrm{dA}_{5}$ tracts is a base pair, for example, exchanged. We expect that the multimers of these oligonucleotides show a similar migration anomaly in the shorter length region where the anomaly is determined by the degree of curvature of the molecules. However, if the sequence modification does change the overall twist angle, the plateau value which is observed for longer molecules should be altered. Since a base pair exchange influences the twist angles to both its neighbors, only relative trinucleotide twist angles can be obtained by this method. However, if nearest neighbor effects dominate the DNA twist angle values, the trinucleotide twist angles might be decomposed into a unique set of sequencespecific dinucleotide twist angles. Thus, in general, experiments as described above should allow us to determine a relative order of sequence-specific DNA tri- (and possibly di-) nucleotide twist angles.

Such behavior as described above was observed for the multimer distributions of the sequences (i)dCTGAATTCAG and (ii) dCGGAATTCCG [30] (Fig. 5). The sequence element dAATT common to both sequences creates a migration anomaly [31] which can be measured with high accuracy. Within experimental error the anomaly is identical for both sequences up to $130 \mathrm{bp}$; beyond this length the anomalies become different and reach a plateau value at $190 \mathrm{bp}$ with a $k$ factor of 1.28 for sequence (i) and at $230 \mathrm{bp}$ with a $k$-factor of 1.39 for sequence (ii) [30]. This difference in the plateau values is assumed not to be due to a different degree of curvature of these sequences since the curvature inducing sequences themselves (dAATT) as well as their neighboring sequences $(d G, d C)$ are unchanged. Furthermore, the same degree of

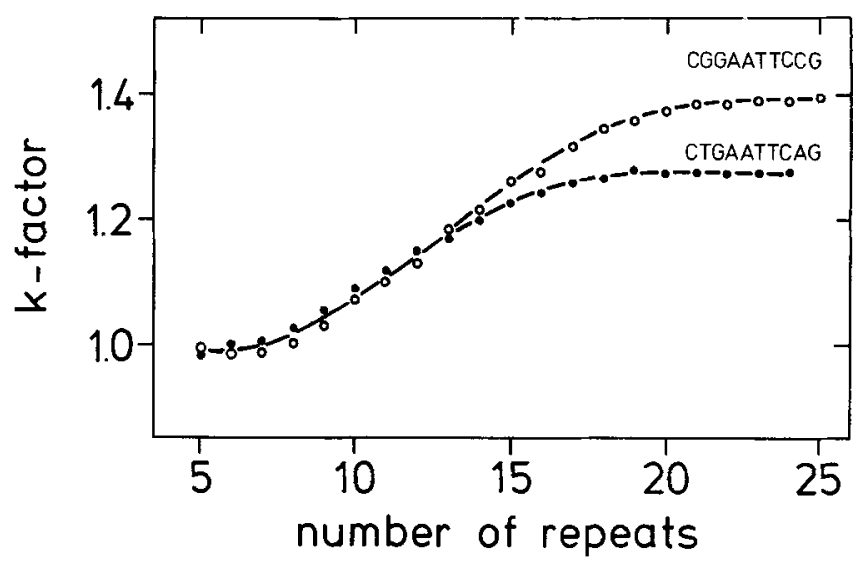

Figure 5. Apparent length divided by sequence length ( $k$-factor) versus the number of repeats for multimers composed of decanucleotides of the sequences 5'-dCGGAATTCCG-3' (O) and 5'-dCTGAATTCAG-3' (O). The $k$-factor is determined in $10 \%$ polyacrylamide gels with HaeIII and Hinfl digests of the plasmid pBR322 [28] as length markers. The experimental error of the $k$-factor is \pm 0.02 (see [30]).

curvature of these sequences is indicated by the identical migration anomaly up to a length of $130 \mathrm{bp}$. Instead, this difference in the plateau values can be attributed to the different twist angle of the sequence dCAGCTG (i) compared to dCCGCCG (ii). Not only is the plateau value of the anomaly different, but also the length at which this plateau is reached. Since the plateau value is higher for the sequence (ii), the data indicate that the sequence repeat of $10 \mathrm{bp}$ is closer to the helical repeat for the sequence (ii) dCGGAATTCCG than for (i) dCTGAATTCAG. The helical repeat of curved sequences was measured to be $10.4 \mathrm{bp} /$ turn [12]. Thus, the helical repeat of sequence (ii) is smaller than that of (i). A smaller helical repeat results from larger twist angles of the local sequence. Thus, the total twist angle of the sequence dCCGCGG should be larger than that of dCAGCTG. Some caution with this in terpretation strictly in terms of twist angles is recommended, since the origin of the plateau effect at longer repeat lengths is not yet understood in detail. It cannot be excluded that variations of wedge angles in the sequence between the dAATT curvature elements contribute to the overall extent of curvature. This contribution cannot be large, however, since the migration anomalies are similar up to repeat lengths of about $130 \mathrm{bp}$.

\subsection{Methyl groups}

The influence of chemical modifications on the DNA helix axis was studied by observing the effect of methyl groups on the DNA. Methylation of DNA sequences is found in vivo in higher and lower organisms (for an overview see [32]). The primary biological function of DNA methylation is assumed to strongly affect sequence-specific interactions of proteins with DNA $[33,34]$. DNA-protein interactions might be influenced by DNA structure changes induced by methylation; local structural variations might be markers for DNA-protein recognition. Self-complementary linkers, $10 \mathrm{bp}$ long, of the sequences dCGGAATTCCG, dCGGGGATCCCG, and dCCATCGATGG were ligated to multimer distributions and analyzed in polyacrylamide gels [35]. The central regions of these sequences are recognized by methylases which methylate the central dAs (EcoRI, dam, and ClaI methylases) or the 
central dCs (BamHI methylase). The mobility of the methylated sequences was measured in the same gels as the unmethylated sequences. A strong enhancement of the migration anomaly (interpreted as DNA curvature) was found for the $E c o$ RI sequence (Fig. 6), a slight anomaly was induced for the BamHI sequence, but no influence on the $C l a I$ sequence was observed [35]. Thus, curvature is not a general property of methylated DNA sequences. Curvature of methylated sites might be an additional DNA-protein recognition feature of some DNA sequences; however, such a creation of DNA curvature is not a general phenomenon. A detailed study of the influence of pyrimidine methyl groups on DNA curvature is in progress (S. Diekmann, L. W. McLaughlin, E. von Kitzing, and A. A. Travers; unpublished). Other chemical modifications, such as the variation of exocy clic substituents of purines, have als been studied using this technique [36]. In general, the influence of chemical base modifications on the DNA structure can be detected by this gel assay. The DNA structure variations may be very small. Such small structure modulations are difficult to observe with other techniques.

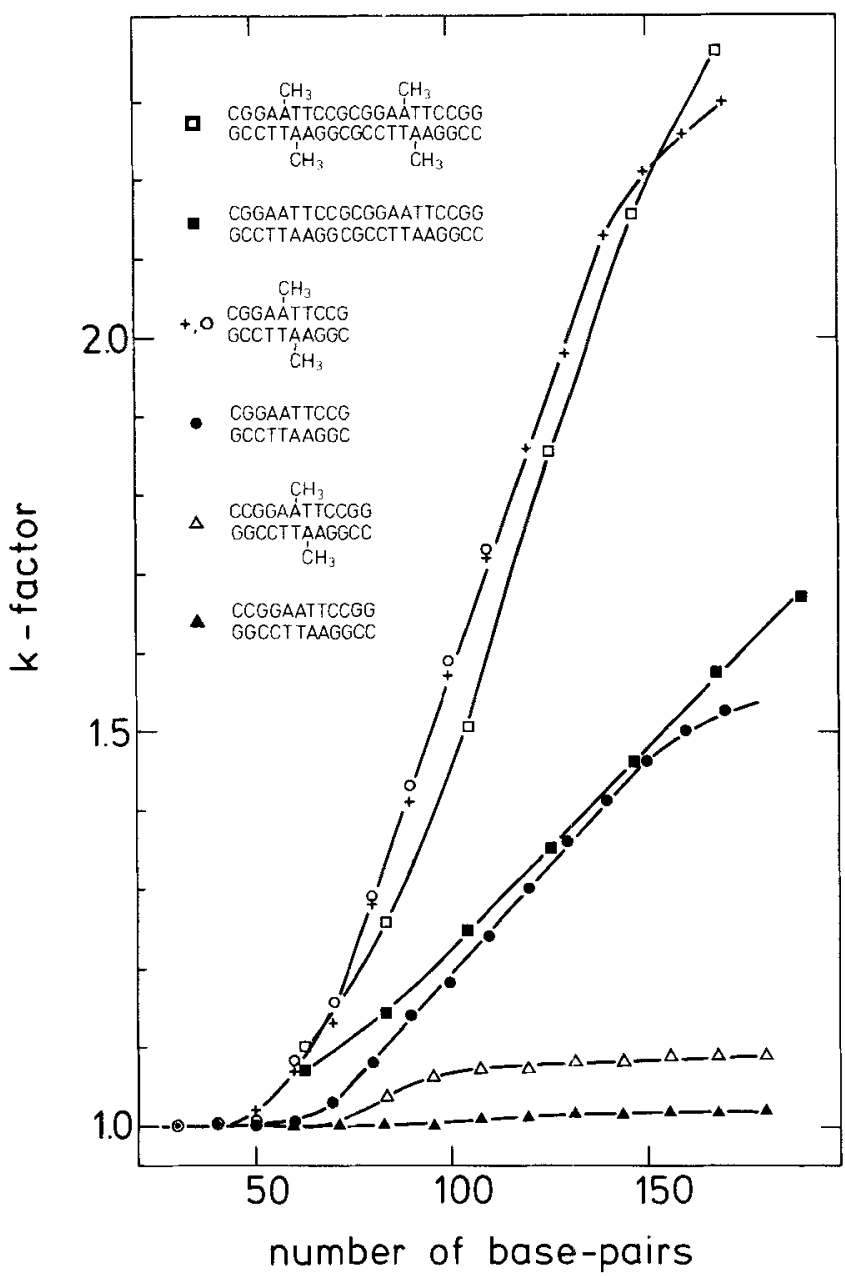

Figure 6. $k$-Factor versus number of base pairs of $10 \mathrm{bp}, 12 \mathrm{bp}$, and $21 \mathrm{bp}$ Eco RI linker multimers determined in a $10 \%$ polyacrylamide gel. (O) 10 bp unmethylated, $(+) 10$ bp methylated by EcoRI methylase and cut by EcoRI, (O) 10 bp methylated by EcoRI methylase but not cut with EcoRI endonuclease, $(\Delta) 12$ bp unmethylated, $(\triangle) 12$ bp methylated by $E c o R I$ methylase and cut by EcoRI, ( $\square$ ) 21 bp unmethyllated, ( $\square$ ) 21 bp methylated by $E c o$ RI methylase and cut by EcoRI. The experimental error of the $k$-factor is \pm 0.02 (see $|35|$ ).

\subsection{Dinucleotide wedge angles}

The effect of gel migration anomaly has been used to measure the influence of single dAA dinucleotides on DNA curvature. If single dinucleotides have a non-zero wedge angle, the influcence of this wedge angle on the DNA curvature is too small to be detected by this gel assay. Ten bp oligonucleotides containing a single dAA dinucleotide do not exhibit migration anomaly [14]. The gel matrix is insensitive to such small degrees of curvature $[20,21]$. Nevertheless, the effect of the small wedge angle could be measured using the described approach: A series of double-stranded oligonucleotides, 21 bp long, having the following sequences, were constructed [29]

\section{5'-dCAAAAACCGTGCCGCCCTCGA-3' \\ 5'-dCAAAAACCGTGCCACCCTCGA-3' \\ 5'-dCAAAAACCGTGCCAACCTCGA-3' \\ $5^{\prime}$-dCAAAAACCGTGCAAACCTCGA-3' \\ 5'-dCAAAAACCGTGCAAAACTCGA-3'}

These 21 mers were ligated to multimer distributions and analyzed in polyacrylamide gels [29] (S. Diekmann, J. Ott, and L. Ulanovsky, unpublished). The $5^{\prime} 10$ bp each contain a $\mathrm{dA}_{5}$ tract which creates a considerable migration anomaly. The other $11 \mathrm{bp}$ contain the sequence of interest. The anomaly is unchanged when going from $\mathrm{dCGC}$ to $\mathrm{dCAC}$ (sequence 0 to 1, Fig. 7). However, a clear increase in $k$-factor is observed for $\mathrm{dCAAC}$ (sequence 2) and a further increase for $\mathrm{dCAAAC}$ (sequence 3 ). The increase in migration anomaly is linear from sequence 1 to 3 , suggesting an additive contribution from both $\mathrm{dAA}$ dinucleotides in sequence 3 . A clear deviation from this linearity is observed for dCAAAAC (sequence 4, Fig. 7). The effect of the three dAA dinucleotides is larger than would be

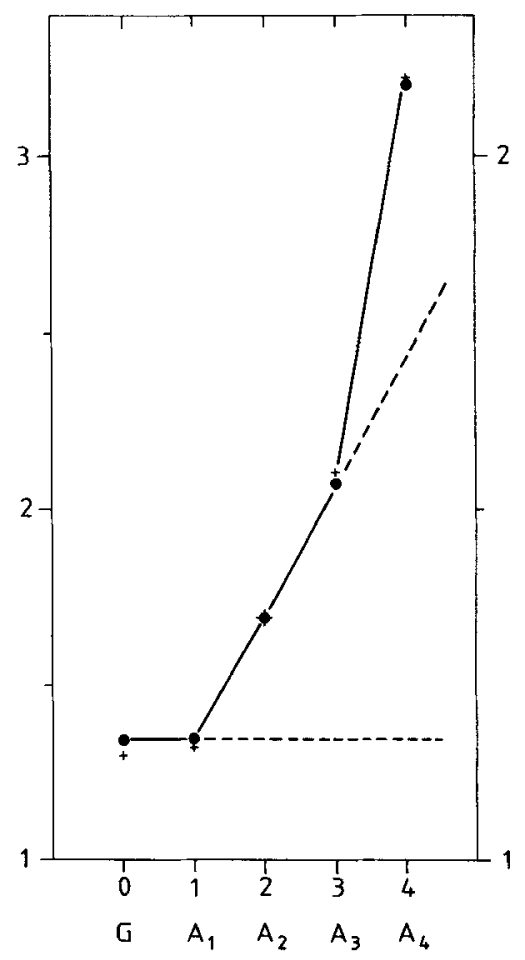

Figure 7. $k$-Factor of $231 \mathrm{bp}(\bullet)$ and $168 \mathrm{bp}(+)$ long fragments of the sequences $5^{\prime}$-dCAAAAACCGTGCXXXXCTCGA-3' with XXXX being $\mathrm{dCGCC}, \mathrm{dCACC}, \mathrm{dCAAC}, \mathrm{dAAAC}$, and $\mathrm{dAAAA}$ plotted versus the number of dAs in the XXXX sequence stretch. The $k$-factor is determined in $10 \%$ polyacrylamide gels with HaeIII and Hinfl digests of the plasmid pBR322 [28] as length markers. The experimental error of the $k$-factor $( \pm 0.02)$ is smaller than the data points (see [29]). 
expected from the additive effects of the two dAA dinucleotides, suggesting a cooperative structural transition between $\mathrm{dA}_{3}$ and $\mathrm{dA}_{4}$.

The migration anomaly (presented as a $k$-factor) shows an approximate quadratic dependence on the degree of curvature of the molecules [20,21]. The $k$-factors displayed in Fig. 7 can be used to estimate the dependence of the curvature per $10 \mathrm{bp}$ of these molecules on the length of the second dA-run [30]. On this basis the curvature depends almost linearly on the number of $\mathrm{dA}$ 's between $\mathrm{dA}_{1}$ and $\mathrm{dA}_{3}$, similar to the $k$-factor dependence. A clear deviation from this line is still evident for $\mathrm{dA}_{4}$. The differences in the migration anomalies for these sequences appear in the whole length range of the multimer distribution (this is displayed for two fragment lengths in Fig. 7). Since anomaly differences are also observed for short multimer lengths, these differences are assumed to be mainly due to different wedge angles of the varied sequences. As discussed above, influences of the twist angles are not expected to affect the anomaly of the shorter molecules, but the levelling-off behavior of the multimer distributions. For 21 mers the sequence repeat matches the helical repeat rather well. As a consequence [22], the $k$-factor does not level off in the observed length range (data not shown).

\subsection{General case}

A migration anomaly in polyacrylamide gels is observed for DNA fragments when they contain a curvature-inducing sequence repeated in phase with the turn of the helix. The value of the effect depends (i) on the degree of curvature of the sequence stretch and (ii) a good phase relation between this sequence repeat and its helical repeat. Both of these properties can be used to measure sequence-specific structural parameters of DNA. While DNA curvature is assumed to be determined by tilt and roll angles between particular base pairs [ 2,3 , $15,17,29]$, the phase relation is directly related to the helical repeat which is given by the dinucleotide twist angles. In general, both sequence-specific structural properties will determine the migration anomaly. For some cases these two influences on the anomaly can be separated. The influence of different dinucleotide twist angles on the migration anomaly may become detectable when, in a repeated sequence of 10 or $11 \mathrm{bp}$ length, those base pairs are changed which are outside an (unchanged) curvature-inducing sequence. The 10 of $11 \mathrm{bp}$ oligonucleotide length closely but not perfectly matches the helical repeat. Thus for these repeat lengths, the anomaly levels off at about $200 \mathrm{bp}$. Only the plateau values would be expected to change by varying the base pair type while the anomaly of shorter multimers should be unchanged. Using this approach we should be able to obtain a relative order of sequence-specific DNA twist angles. The gel matrix is not sensitive to small degrees of curvature as they are induced within the sequence by particular dinucleotide wedge angles. The assay, however, becomes very sensitive when such a sequence of interest alternates with a considerably curved sequence stretch in phase with the helix turn. When the curvature-inducing sequence itself is varied and, thus, tilt and roll angles are modified, the migration anomaly should be different also for the shorter multimers in the distribution. In this way we should be able to get a measure of the relative order of sequence-specific DNA wedge angles.

\section{References}

[1] Dickerson, R.E. and Drew, H. R.,J.Mol.Biol. 1981, 149,761-786.

[2] Trifonov, E. N., CRC Crit. Rev. Biochem., 1986, 19, 89-106.

[3] Diekmann, S., in: Eckstein, F, and Lilley, D.(Eds.), Nucleic Acids and Molecular Biology, Springer, Berlin 1987, pp. 138-156

[4] Ulanovsky, L., Bodner, M., Trifonov, E. N. and Choder, M., Proc. Natl. Acad. Sci. USA 1986, 8, 862-866.

[5] Levene,S.D. and Crothers, D. M.,J.Mol.Biol. 1986,189,61-72.

[6] Griffith, J., Bleyman, M., Rauch, C. A., Kitchin, P. A. and Englund, P. T., Cell 1986, 46, 717-724.

[7] Theveny, B., Coulaud, D., LeBret, M. and Revet, B., in: Olson, W. K. Sarma, M. H., Sarma, R. H. and Sundaralingam, M.(Eds.), Structure and Expression, Volume 3 of DNA Bending and Curvature, Adenine Press, Schenectady 1988, pp. 39-55.

[8] Marini, J. C., Levene, S. D., Crothers, D. M. and Englund, P. T., Proc. Natl. Acad. Sci. USA 1982, 79, 7664-7668.

[9] Marini, J. C. and Englund, P. T. Proc. Natl.Acad. Sci. USA 1983, 80, 7678.

[10] Wu, H. M. and Crothers, D. M., Nature 1984, 308, 509-513.

[11] Hagerman, P. J., Biochemistry 1985, 24, 7033-7037.

[12] Diekmann, S. and Wang, J. C., J. Mol. Biol. 1985, 186, 1-11.

[13] Hagerman, P. J., Nature 1986, 321, 449-450.

[14] Diekmann, S., FEBS Let. 1986, 195, 53-56.

[15] Koo, H. S., Wu, H. M. and Crothers, D. M., Nature 1986, 320, 501-506.

[16] Sarma, M. H., Gupta, G. and Darma, R. H., Biochemistry 1988, 27, 3423-3432.

[17] Nelson, H. C. M., Finch, J. T., Luisi, B. F. and Klug, A., Nature 1987, 330, 221-226.

[18] Coll, M., Frederick, C. A., Wang, A. H.-H. and Rich, A., Proc. Natl. Acad. Sci. USA 1987, 84, 8385-8389.

[19] Stellwagen, N. C., Biochemistry 1983, 22, 6186-6193.

[20] Trifonov, E. N. and Ulanovsky, L., in: Wells, R. D. and Harvey, S.C. (Eds.), Unusual DNA Structures, Springer, New York 1988, pp. 173-187.

[211 Koo, H.S. and Crothers, D. M., Proc. Natl.Acad.Sci. USA 1988,85, 1763-1767.

[22] Calladine, C. R., Drew, H. R. and McCall, M. J., J. Mol. Biol. 1988, 201, 127-137.

[23] Nishimura, H., Poster presentation at the EMBO workshop on DNA Curvature and Bending in Cambridge, England, September 1988.

[24] Diekmann, S. and Pörschke, D., Biophys. Chem. 1987, 26, 207-216.

[25] Ott, J. and Eckstein, F., Nucleic Acids Res. 1984, 23, 9137-9142.

[26] Maxam, A. M. and Gilbert, W., Methods Enzymol. 1980, 65, 499-560.

[27] Diekmann, S., Nucleic Acids Res. 1987, 15, 247-265.

[28] Sutcliffe, J. G., Cold Spring Harb. Symp. Quant. Biol. 1979, 43, 77-90.

[29] Diekmann, S. and von Kitzing, E., in: Olson, W. K., Sarma, M. H., Sarma, R. H. and Sundaralingam, M. (Eds.), Structure and Expression, Volume 3 of DNA Bending and Curvature, Adenine Press, Schenectady 1988 , pp. 57-67.

[30] Diekmann, S. and McLaughlin, L. W., J. Mol. Biol. 1988, 202, 823-834.

[31] Hagerman, P. J., in: Wells, R. D. and Harvey, S. C. (Eds.), Unusual DNA Structures, Springer, New York 1988, pp. 196-207.

[32] Razin, R., Cedar, H. and Riggs, A. D., in: Razin, R., Cedar, H. and Riggs, A. D. (Eds.) DNA Methylation, Springer, Berlin 1984, pp. 1-10.

[33] Engel, J. D. and von Hippel, P. H.,J. Biol.Chem. 1978, 253,927-934.

[34] Razin, A. and Riggs, A. D., Science 1980, 210, 604-610.

[35] Diekmann, S., EMBO J. 1987, 6, 4313-4217.

[36] Diekmann, S., von Kitzing, E., McLaughlin, L., Ott, J. and Eckstein, F., Proc. Natl. Acad. Sci. USA 1987, 84, 8257-8262.

[37] Diekman, S. and Lilley, D. M. J., Nucleic Acids Res. 1987, 15, $5765-5774$. 